\title{
Concordancia diagnóstica intraoperador de dos métodos cuantitativos de necesidad de tratamiento ortodóncico en niños
}

\section{Intraoperative diagnostic concordance of two quantitative methods of need for orthodontic treatment in children}

\begin{abstract}
Resumen
Objetivo. El presente estudio tuvo como objetivo evaluar la concordancia diagnóstica intraoperador, aplicando dos métodos cuantitativos como lo son el índice estético dental $(D A I)$ y el índice de necesidad de tratamiento ortodóncico (IONT) en niños con dentición mixta. Métodos. Se realizó un estudio observacional correlacional de corte transversal, con una muestra seleccionada de 20 pacientes que concurrieron para su atención integral al Módulo de atención integral del niño y el adolescente de la Facultad de Odontología, Universidad Nacional del Nordeste (FOUNNE). Sobre los mismos pacientes se midieron las variables incluidas en ambos índices en una primera medición y con una diferencia de tiempo de 15 días una segunda medición, para evaluar la concordancia diagnóstica intraoperador y para evitar el sesgo por la memoria de este. Resultados. Se realizó un análisis de correlación de Spearman, encontrándose para el IONT un coeficiente $r=1$ lo que indica una correlación perfecta y para el DAI un $\mathrm{r}=0,99$, ambos significativos. Conclusiones. Se comprobó una correcta concordancia diagnóstica intraoperador de necesidad de tratamiento ortodóncico en la aplicación de dos métodos cuantitativos, el índice estético dental y el índice de necesidad de tratamiento, sobre los mismos niños con dentición mixta.
\end{abstract}

Palabras clave: Índice; Oclusión dental; Ortodoncia (fuente: DeCS BIREME).

\section{Artículo Original}

Paola Berenice Olivera ${ }^{1, a}$, Edna Yohana Meza ${ }^{1, a}$, María Silvina Dho ${ }^{1, b}$, Alina Noelia Peláez ${ }^{1, b}$

\author{
${ }^{1}$ Universidad Nacional del Nordeste, Facultad de Odonto- \\ logía, Corrientes, Argentina. \\ a Cirujano-dentista. \\ ${ }^{\text {b }}$ Doctor en Odontología.
}

\section{Correspondencia:}

Paola Berenice Olivera: paolaolivera27@gmail.com Facultad de Odontología. Universidad Nacional del Nordeste. FOUNNE. Avenida Libertad 5450. Corrientes Argentina. C.P. 3400.

ORCID: 0000-0003-0175-9919

\section{Coautores:}

Edna Yohana Meza: yohanameza.2@gmail.com ORCID: 0000-0002-8636-7254

María Silvina Dho: silvinadho@gmail.com ORCID: 0000-0001-9374-2027

Alina Noelia Peláez: anpelaez@odn.unne.edu.ar ORCID: 0000-0002-2715-8524

\section{Editora:}

María Antonieta Pérez Flores Universidad de Concepción, Chile.

Conflicto de intereses: los autores declaran no tener conflictos de interés.

Fuente de financiamiento: proyecto acreditado PI $\mathrm{N}^{\circ} \mathrm{J}-002$, financiado por la Secretaría General de Ciencia y Técnica de la Universidad Nacional del Nordeste.

Recibido: 02/10/19

Aceptado: 18/01/20

Publicado: $24 / 02 / 20$ 
was performed, finding for the IONT a coefficient $\mathrm{r}=1$ indicating a perfect correlation and for the DAI a r $=0.99$, both significant. Conclusions. A correct intraoperative diagnostic concordance of the need for orthodontic treatment was verified in the application of two quantitative methods, the dental aesthetic index and the treatment necessity index, on the same children with mixed dentition.

Keywords: Index; Dental occlusion; Orthodontics (Source: MeSH NLM).

\section{Introducción}

Las maloclusiones son un término universalmente aceptado, pero no se puede considerar como lo opuesto a una oclusión normal. Generalmente alguna desviación en la oclusión es calificada como maloclusión, y aquí surge el posible compromiso de aceptar como único normal, lo ideal ${ }^{1}$. El término maloclusión es genérico y debe aplicarse, sobre todo, a aquellas situaciones que requieren de algún tratamiento ortodóncico, más que a cualquier desviación de la oclusión normal. La calificación de normal o anormal es una cuestión de grados que debe ser medida individualmente en cada caso ${ }^{2}$.

Existe una alta prevalencia de maloclusiones (MO) a nivel mundial y según la Organización mundial de salud (OMS) las MO ocupan el tercer lugar entre los problemas bucales más frecuentes ${ }^{3}$. Es por ello que al no existir un criterio uniforme de lo que se considera o no maloclusión, tampoco existe acuerdo a la hora de decidir cuándo empiezan las necesidades de tratamiento ortodóncico. Si bien se han creado un gran número de índices epidemiológicos para el diagnóstico de la necesidad de tratamiento ortodóncico, ninguno ha sido considerado universal ${ }^{1}$.

Los métodos cualitativos de valoración de las maloclusiones como instrumentos epidemiológicos, no se realizan con frecuencia actualmente, ya que poseen limitaciones a la hora de poder determinar el grado y severidad de maloclusión. Además, se considera poco objetivo, por lo que no se pueden extrapolar los resultados a la población. En cambio, clínicamente la aplicación de la clasificación de Angle, sigue siendo una de las más aceptadas y utilizadas en la actualidad ${ }^{4}$.

Disponer de métodos cuantitativos como herramientas epidemiológicas orales resulta fundamental para poder valorar de forma objetiva la severidad y grado de maloclusión junto con la necesidad de tratamiento ortodóncico. Es por ello, que se recurre a la utilización de índices oclusales. Los más utilizados en la actualidad son el índice estético dental, conocido por sus siglas en inglés como dental aesthetic index $(D A I)$ y el índice de necesidad de tratamiento ortodóncico, de su traducción al inglés: index of orthodontic treatment need (IOTN) ${ }^{5}$.

La concordancia diagnóstica se puede definir como el grado de conformidad entre dos variables, en la evaluación de la misma unidad de análisis. Radica su importancia cuando se utiliza para comprobar la factibilidad y confiabilidad de un instrumento a la hora de identificar posibles casos o confirmar la presencia de trastornos en la oclusión ${ }^{6}$. Se la considera como un paso fundamental para realizar una investigación científica adecuada, ya que en cada toma de registro se aplican criterios que llevan al diagnóstico de la patología o trastorno en cuestión y es necesario constatar si el grado de certeza diagnóstica es el correcto. Es por ello que, el presente estudio se realiza para brindar información acerca de la evaluación de la concordancia diagnóstica intraoperador para evitar errores, ya que todos los instrumentos y el mismo proceso de toma de registro lleva algún grado de error que se debe conocer y asumir.

Si bien son índices validados para su utilización en adultos, algunos autores lo han probado en niños ${ }^{7}$ y dada la necesidad de medir el grado de maloclusión y la necesidad de tratamiento ortodóncico en niños que concurren al Módulo de atención integral del niño y el adolescente en la Facultad de Odontología de la Universidad Nacional del Nordeste, Corrientes, Argentina, se ha realizado la presente investigación, cuyo objetivo fue evaluar la concordancia diagnóstica intraoperador, aplicando dos métodos cuantitativos como lo son el índice estético dental $(D A I)$ y el índice de necesidad de tratamiento ortodóncico $(I O T N)$ en niños con dentición mixta.

\section{Métodos}

Se realizó un estudio observacional correlacional de corte transversal. La población de estudio estuvo constituida por todos los pacientes que asistieron a la Clínica del Módulo Atención Integral del Niño y Adolescente de la Facultad de Odontología de la UNNE, para su atención integral en el período del mes de junio del año 2017. Se aplicaron los siguientes criterios de inclusión: pacientes con dentición mixta entre 6 y 12 ańos, pacientes, padres y/o tutores que aceptaron formar parte del estudio disponiendo de su consentimiento para la utilización de los datos de exploración. Los criterios de exclusión fueron: pacientes que presentaron alguna patología sistémica significativa con deficiencia motriz o neurológica y aquellos pacientes que habían recibido o estaban recibiendo en ese momento, cualquier tipo de tratamiento ortopédico u ortodóncico.

En base a estos criterios se seleccionaron 20 pacientes a través de un muestreo al azar sistemático, a los cuales se les realizó el $D A I$ y el $I O T N$. Se midieron las variables incluidas en ambos índices en una primera medición y con una diferencia de tiempo de 15 días se volvió a medir los índices oclusales, con el objetivo de evitar el sesgo por la memoria de mismo.

El examen clínico del paciente se llevó a cabo en las Clínicas de la Facultad de Odontología de la Universidad 
Nacional del Nordeste. Primero, se les presentó una hoja informativa a los pacientes, padres y/o tutores de los procedimientos y objetivos del trabajo quienes posteriormente firmaron un consentimiento informado. El examen clínico se realizó en sillones dentales, los cuales fueron efectuados por un único examinador entrenado para tal efecto. El operador cuenta con experiencia clínica desde el año 2013, en el marco de Proyectos de Investigación acreditados por la Universidad Nacional del Nordeste, en la medición y observación de las variables de métodos cuantitativos para el diagnóstico de necesidad de tratamiento ortodóncico.

A todos los pacientes que conformaron la muestra de estudio se les aplicó el $D A I$, las variables del mismo son expresadas en milímetros las cuales fueron tomadas con sonda milimetrada William-Fox (Hu-Friedy) instrumento previamente validado ${ }^{8}$, la metodología que se utilizó para el cálculo del $D A I$, de cada paciente y los criterios de recogida de datos, corresponden a los preconizados por la OMS, según el Manual de Levantamiento Epidemiológico Básico de Salud Bucal ${ }^{9}$.

Las diez variables y parámetros con sus correspondientes coeficientes de ponderación utilizados por el índice son el número de dientes ausentes, apiñamiento en el sector anterior, espaciamiento en el sector anterior de la cavidad bucal, la presencia de diastemas en la línea media, la irregularidad tanto del maxilar superior como en la mandíbula, el resalte maxilar y resalte mandibular, la presencia de mordida abierta y por último la relación molar anteroposterior.

Una vez obtenidos los valores correspondientes de las diez variables del DAI de cada paciente, se realizó la ecuación (Figura 1) multiplicando dichos valores con sus coeficientes de ponderación más 13 que es el valor constante del índice. Se sumaron los totales y se obtuvo una puntuación global final, la cual permite identificar al paciente en el intervalo de ponderación, según su severidad de maloclusión y el grado de necesidad de tratamiento ortodóncico. Si el puntaje fue menor o igual a 25 , se consideró una oclusión normal. Si el puntaje final fue de 26 a 30 puntos, se consideró una maloclusión manifiesta y el tratamiento ortodóncico era optativo. $\mathrm{Si}$ el puntaje era de 31 a 35 puntos, se consideró como una maloclusión severa y el tratamiento es muy deseable. Si el puntaje superaba los 36 puntos, era considerado una oclusión muy severa o discapacitante y el tratamiento ortodóncico es obligatorio.
A los mismos pacientes también se les realizó el IOTN, en los que se evaluaron las cinco categorías o grados de necesidad de tratamiento. Estas mediciones se realizaron también con la ayuda de una sonda milimetrada ${ }^{8}$. A diferencia del anterior índice, a este no se le asignaron puntuaciones, ni se obtuvo una puntuación numérica global, sino que el rasgo más severo identificado al examinar al paciente es lo que sirvió de referencia para incluirlo dentro de un grado u otro. Cada rasgo oclusal identificado se clasificó en uno de los cinco grados según la contribución relativa de cada uno, ya que el IOTN estipula puntos de corte claros entre cada nivel.

Para la evaluación de la concordancia diagnóstica se tuvieron en cuenta las variables correspondientes a los valores finales arrojados por los índices, en sus dos tomas con la diferencia de tiempo de 15 días. Se analizaron las cuatro categorías del DAI y los cinco grados del IOTN. Una vez que se calcularon los valores finales cada paciente, se correlacionaron las variables de los resultados del $D A I$ de la primera toma, con los resultados finales del $D A I$ de la segunda toma. De la misma manera se procedió con el IOTN.

Se realizó un análisis descriptivo de los datos y para evaluar la concordancia diagnóstica intraoperador se utilizó el análisis de correlación de Spearman. El análisis de los datos se hizo mediante el software estadístico InfoStat versión $2018{ }^{10}$.

\section{Resultados}

Los pacientes evaluados tenían un rango de edad de 6 a 11 ańos, con un promedio de 7,45 $\pm 1,19$ ańos. De los 20 pacientes evaluados el 55\% correspondió al sexo femenino y un $45 \%$ al sexo masculino.

Al total de la muestra seleccionada se le realizó el $D A I$, de los cuales se puede observar que el 40\% (n: 8) presentó una oclusión normal sin necesidad de realizarse tratamiento ortodóncico, el 35\% (n: 7) demostró tener una maloclusión discapacitante con una necesidad obligatoria de tratamiento ortodóncico, el 15\% (n: 3) de los pacientes presento una maloclusión severa con necesidad de tratamiento muy deseable, mientras que el 10\% (n: 2) de la población tuvo una maloclusión manifiesta con necesidad ortodóncica optativa.

A los mismos pacientes también se les midió el IOTN, se determinó que el 50\% (n: 10) presentaron Grado 1 que incluye una oclusión normal por lo cual no necesita

\section{ECUACIÓN DAI: \\ Ausencia (x 6) + Apiñamiento + Espaciamiento + Diastema (x 3) + Irregularidad superior + Irregularidad inferior + Resalte maxilar (x 2) + Resalte mandibular (x 4) + Mordida abierta anterior (x 4) + Relación molar anteroposterior $(x 3)+13$ = Índice estético dental.}

Figura 1. Ecuación del índice estético dental (DAI) 
tratamiento ortodóncico. El 25\% (n: 5) presentó Grado 2 que padecen de una maloclusión leve que apenas necesita tratamiento, y finalmente un $15 \%$ (n: 3 ) presento Grado 4 con una maloclusión grave con necesidad de tratamiento ortodóncico. El Grado 3, maloclusiones moderadas con necesidad dudosa de tratamiento y el Grado 5, con maloclusiones extremas con una necesidad obligatoria de tratamiento, presentaron un $5 \%$ en ambos casos.

Para poder evaluar la concordancia diagnóstica intraoperador, se realizó la prueba de correlación de Spearman, encontrándose para el IONT un coeficiente r=1 lo que indica una correlación perfecta (Figura 2) y para el $D A I$ un $\mathrm{r}=0,98$ (Figura 3) ambos significativos. Esto demuestra la correcta calibración del operador que, aplicados sobre los mismos pacientes con diferencia de 15 días, arrojaron los mismos resultados.

\section{Discusion}

En este trabajo se propuso evaluar la concordancia diagnóstica por parte del operador, de los valores obtenidos mediante la utilización de dos índices epidemiológicos, como lo son el DAI y el IOTN, que son los métodos más utilizados mundialmente.

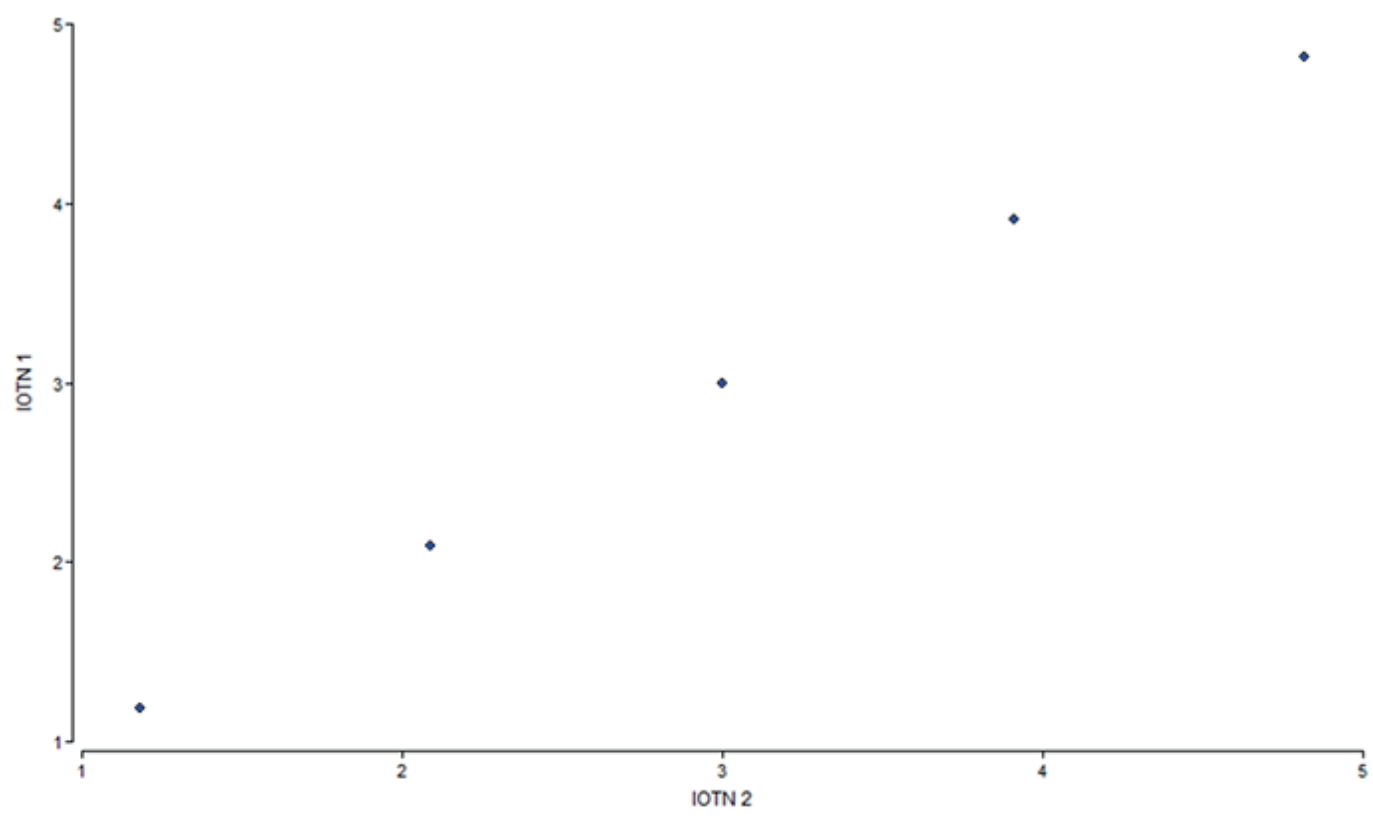

Figura 2. Correlación de Spearman para la evaluación diagnóstica intraoperador del índice de necesidad de tratamiento ortodóncico (IOTN)

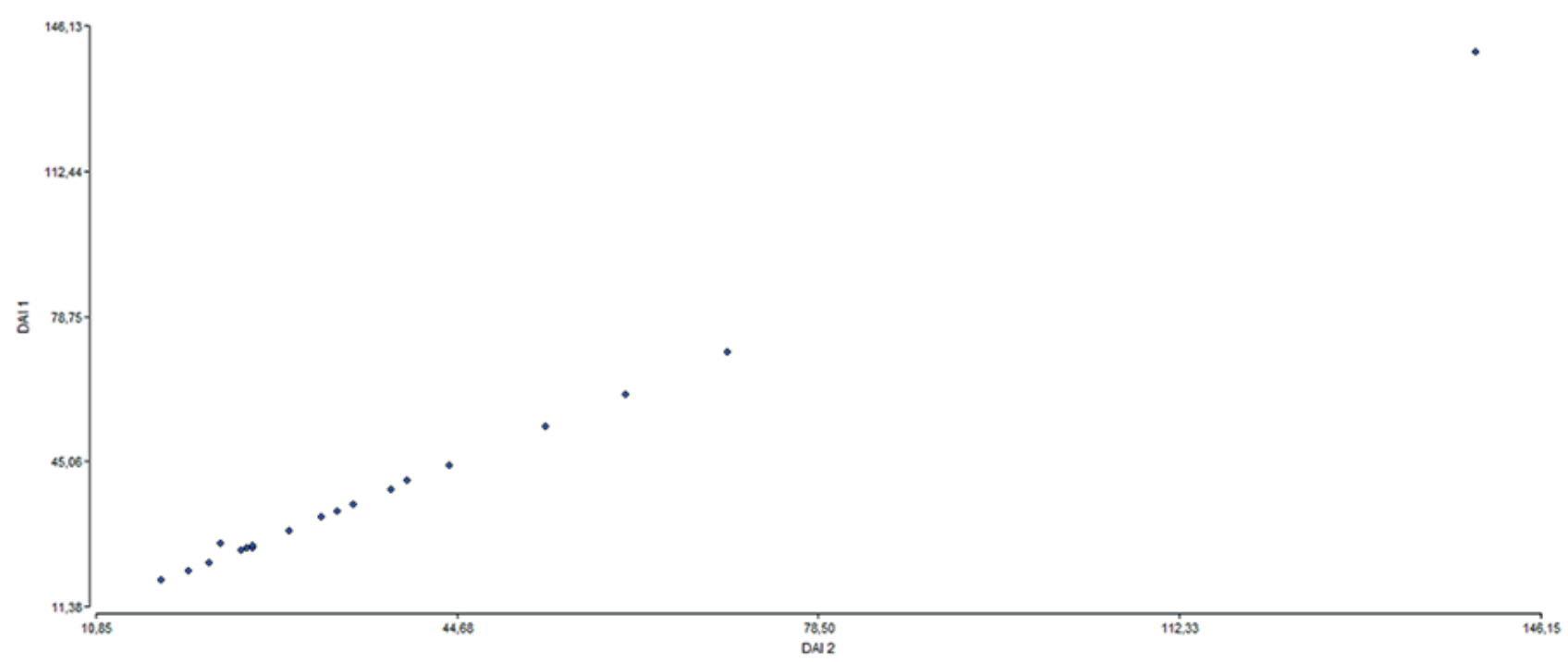

Figura 3. Correlación de Spearman para la evaluación diagnóstica intraoperador del índice estético dental (DAI) 
Los estudios de concordancia diagnóstica intraoperador, son fundamentales para en estimar hasta qué punto un único observador coincide en su medición sobre una misma unidad de análisis, con una diferencia de tiempo. Se consideran esenciales para poder medir la confiabilidad o reproducibilidad de las evaluaciones, en las investigaciones epidemiológicas. Se dice que un procedimiento es preciso, si sus resultados son consistentes cuando se aplica en dos oportunidades, bajo las mismas circunstancias, al mismo individuo. La precisión de un procedimiento se ve afectada por dos factores fundamentales, la variación propia del instrumento o procedimiento y la variación del examinador. La primera de ellas tiene que ver con la calidad y la calibración del instrumental de medida y diagnóstico. La variación del examinador está relacionada con su entrenamiento, formación y capacidad, y también se llama error del examinador. A menor variación de éste, mayor precisión se consigue a la hora de realizar una prueba y por tanto más válido será el resultado ${ }^{6}$.

La importancia del presente estudio radica en informar acerca de la concordancia intraoperador, independientemente del diseño de la investigación, la validez de un estudio puede verse afectada si se utilizan mediciones poco fiables. En los resultados expuestos anteriormente se puede determinar, que si se toman correctamente los diferentes parámetros de los índices, se puede obtener una correcta calibración y entrenamiento por parte del profesional odontólogo. La ventaja de que sea un único examinador que toma los registros, es que permite documentar las evidencias explícitas sobre el desempeño, para identificar aquellas áreas estudiadas en los distintos aspectos de los índices aplicados en los niños con dentición mixta.

Con relación a esto no existe información en la literatura, por lo que no se puede comparar con otras realidades, aunque sí existen trabajos como el de Cardoso et al. ${ }^{11}$ quienes realizaron su investigación con el objetivo de evaluar la validez y la reproductibilidad del $D A I$ y el $I O T N$ para la identificación de necesidades de tratamiento ortodóntico. En sus resultados, hallaron validez entre ambos índices, del $61 \%$ para el $D A I$ y $67 \%$ para el componente de salud del IOTN.

Guamán Rodríguez ${ }^{12}$ en su investigación sobre la correlación del índice de estética dental y la maloclusión dental para el diagnóstico de tratamientos ortodóntico realizada en 20 modelos de yeso que fueron donados por alumnos del segundo semestre de la carrera de Odontología de la UNACH, se encontró la siguiente concordancia diagnostica: entre dos operadores que evaluaron los 20 modelos estudio, el primer experto determinó que el 5\% de los modelos tenían una maloclusión de grado 1 , el $15 \%$ una maloclusión de grado 2 , el $5 \%$ una maloclusión de grado 3 y el $75 \%$ una maloclusión de grado 4 representando así este último a la mayoría de los modelos, no habiendo concordancia con lo determinado por el segundo experto que, establece que el $20 \%$ de los modelos tienen una maloclusión de grado 1 , el $25 \%$ una maloclusión de grado 2 al igual que el grado 3 y el 30\% una maloclusión de grado 4. Por lo que concluyen que, entre los dos expertos, sí existe una diferencia significativa entre ellos y esto se debe a que tanto el experto 1 como el experto 2 no comparten criterios similares al momento de analizar cada modelo de estudio. En contraposición con los resultados hallados en el presente estudio en el que la evaluación diagnostica fue muy significativa, encontrando $\mathrm{r}=1$ para el $I O T N$ y $\mathrm{r}=0,98$ para el $D A I$.

Araya Vallespir et al. ${ }^{13}$ determinaron el grado de concordancia de los índices más utilizados en estudios epidemiológicos de la enfermedad periodontal, en adultos 35-44 años en Lorenzo Arenas, Concepción, en el año 2013. Aseguran la importancia de unificar los criterios y definir universalmente las alteraciones en la cavidad bucal, situación que queda reflejada al observar cómo la prevalencia varia enormemente al modificar los indicadores utilizados para su medición. Este es uno de los motivos del porqué las comparaciones de los distintos estudios epidemiológicos se ven dificultadas.

Por otra parte, González Sosa ${ }^{14}$, refiere que el error entre los evaluadores aumenta en grandes rasgos, a medida que interfieren en la evaluación las cualidades subjetivas de los investigadores, como son la habilidad y el juicio dados por la experiencia. Concluyen que se debe emplear un solo investigador calificado para el caso. Es debido a estas situaciones que en el presente estudio se utilizó un solo examinador en las mediciones de ambos índices.

Alemán Estévez et al. ${ }^{15}$ en su estudio para determinar la necesidad de tratamiento de Ortodoncia mediante la aplicación del índice de estética dental en 450 estudiantes de 8-9 ańos de edad, del área de salud Milanés, Matanzas, mencionan que hallaron discrepancias en sus resultados, ocasionadas por las maloclusiones que el índice no tiene en cuenta, por lo cual recomiendan que, al utilizarse el $D A I$ en pacientes pediátricos con dentición mixta, se tenga en cuenta el criterio del profesional capacitado para tener mayor grado de confiabilidad a la hora de los resultados.

Coincidiendo con lo expresado por Alemán Estévez et al. ${ }^{15}$, en la presente investigación también se utilizó el $D A I$ en dentición mixta, por lo que se estima que esta diferencia $(r=0,99)$ entre las dos tomas por parte del mismo operador puede deberse a que la población en la que se trabaja son niños con dentición mixta, los que se encuentran en proceso de constante cambio y desarrollo, y el tiempo estimado para evitar la memoria del operador, de 15 días, se pudieron haber producido modificaciones, como por ejemplo la más característica que fue la exfoliación dentaria.

Como conclusión del trabajo se comprobó una correcta concordancia diagnóstica intraoperador de necesidad de tratamiento ortodóncico en la aplicación de dos métodos cuantitativos, el índice estético dental y el índice de necesidad de tratamiento, sobre los mismos niños con dentición mixta. 


\section{Referencias bibliográficas}

1. Cid Gutiérrez CM. Estudio epidemiológico de maloclusiones en niños de 6 a 15 años de la Comunidad de Madrid de acuerdo con el índice estético dental comparación entre dos grupos. [tesis doctoral]. [Madrid]. Facultad De Odontología. Universidad Complutense De Madrid; 2008.

2. Canut Brusda JA. Oclusión normal y maloclusión. Ortodoncia clínica y terapéutica. $2^{\text {a }}$ ed. Barcelona: Masson S.A.; 2000. p. 95-104.

3. Moreno Arias A, Sáez Luna ME, Sotolongo Chacón L, Alegret Rodríguez ML. Validación de un índice para determinar la necesidad y la prioridad del tratamiento ortodóncico. Acta Médica del Centro. 2016;10(2):36-43

4. Pletilla T, Pletilla I, Widsten E, Varrela I, Alanen P. Extent and prevision of orthodontic service for children and adolescents in Finland. Com Dent Oral Epidemiol. 1997;(25):150-5

5. Mercado S, Mercado J, Tapia R. Maloclusiones y calidad de vida en adolescentes. Kiru. 2018;15(2):94-8.

6. Campo-Arias A, Herazo E. Concordancia intra-e interevaluadores. Rev Colomb Psiquiatr. [Internet]. 2010 [citado 2019 julio 03]; 39(2):424-32. Disponible en: http://www.scielo.org.co/scielo.php?script=sci_arttext\&pid=S0034-74502010000200015\&lng=en.

7. Alemán Estévez MG, Martínez Brito I, Pérez Lauzurique A. Necesidad de tratamiento ortodóncico en escolares. Aplicación del índice DAI. Rev Med. Electrón. [Internet]. 2011 [citado 2019 Jun 27]; 33(4):441-7. Disponible en: http://scielo.sld.cu/scielo.php?script=sci_arttext\&pid=S1684-18242011000400006\&lng=es.

8. Peláez AN, Ponce AJ, Mazza SM. Validación de la Sonda Milimetrada como Instrumento de medida del Índice Estético Dental. Odontoestomatología [Internet]. 2013 [citado 2019 Jun 28]; 15(22): 16-23. Disponible en: http://www.scielo.edu.uy/scielo.php?script=sci_arttext\&pid=S1688-93392013000300003\&lng=es.
9. OMS. Encuestas de salud Bucodental. Métodos Básicos. Organización Mundial de la Salud. 4 ed. Ginebra. 1997. p 46-52.

10. Di Rienzo JA, Casanoves F, Balzarini MG, Gonzalez L, Tablada M, Robledo CW. InfoStat versión 2018. Grupo InfoStat, FCA, Universidad Nacional de Córdoba, Argentina. URL http://www.infostat.com.ar.

11. Cardoso CF, Drummond AF, Lages EM, Pretti H, Ferreira EF, Abreu MH. The dental aesthetic index and dental health component of the index of orthodontic treatment need as tools in epidemiological studies. Int J Environ Res Public Health. [Internet] 2011 [citado 28 Jun 2019]; 8(8):3277-86. Disponible en: http://www.ncbi.nlm.nih. gov/pubmed/21909306

12. Guamán Rodríguez GS. Correlación del índice de estética dental y la mala oclusión dental para el diagnóstico de tratamientos ortodónticos. [Proyecto de investigación]. [Ecuador]. Facultad De Odontología. Universidad Nacional de Chimborazo, 2019. Jun [citado 2019 Jul 06]; Disponible en: http://dspace.unach.edu.ec/handle/51000/5582

13. Araya Vallespir C, Ortega CU, Machuca LL, Rodriguez Vera M, Silva Contreras S. Grado de concordancia de los índices más utilizados en estudios epidemiológicos de la enfermedad periodontal. Rev Clin Period, Implantol Rehab Oral. 2014;7.(3):175-9.

14. González Sosa G. Aplicación del método clínico para el diagnóstico de pacientes con hemorragia digestiva alta no variceal. Rev cubana Med Gen Integr [Internet]. 2016 [citado 2019 Jul 05]; 32(2):258-67. Disponible en: http://scielo.sld.cu/scielo.php?script=sci_arttext\&pid=S086421252016000200013\&lng=es.

15. Alemán Estévez MG, Martínez Brito I, Pérez Lauzurique A. Índice de estética dental y criterio profesional para determinar la necesidad de tratamiento ortodóntico. Rev Med Electrón. [Internet]. 2011 [citado 2019 Jun 28]; 33(3):271-7.Disponible en: http://scielo.sld.cu/scielo.php?script=sci_arttext\&pid=S168418242011000300003\&lng=es. 